\title{
Multicenter Evaluation of Emergency Department Treatment for Children and Adolescents With Crohn's Disease According to Race/Ethnicity and Insurance Payor Status
}

\author{
Jennifer L. Dotson, $M D, M P H_{,}^{*,+}$ Michael D. Kappelman, $M D, M P H_{,}^{\neq}$Josh Bricker, $P h D_{,}^{+}$ \\ Rebecca Andridge, $\mathrm{PhD},{ }^{\S}$ Deena J. Chisolm, PhD, ${ }^{+}$and Wallace V. Crandall, MD, MMM*
}

\begin{abstract}
Background: Racial and socioeconomic disparities exist in the treatment and outcomes of children and adults with Crohn's disease (CD). This study investigated the impact of race and insurance status on emergency department (ED) evaluation and treatment among children with CD in the United States.

Methods: Data from the Pediatric Health Information System included ED visits between January 2007 and December 2013 for patients aged $\leq 21$ years with a primary diagnosis of $\mathrm{CD}$, or a secondary diagnosis of $\mathrm{CD}$ plus a primary CD-related diagnosis. Analyses were performed using mixed-effects logistic regression.

Results: Subjects included 2618 unique patients (black, 612 [23\%]; white, 2006 [77\%]) with 3779 visits from 38 hospitals, a median age of $14.0 \pm 4.0$ years, and $50 \%$ male. White children had a higher median neighborhood income and were more likely to have private insurance $(57 \%$ vs $30 \% ; P<0.001$ ). Emergency department visits for privately insured patients had higher odds of complete blood count (odds ratio [OR], 1.43; $95 \%$ CI, 1.08-1.90) and C-reactive protein/erythrocyte sedimentation rate (OR, 1.39; 95\% CI, 1.06-1.82) vs Medicaid insured. Visits for white children had higher odds of receiving antiemetics (OR, 1.52; 95\% CI, 1.06-2.17) vs black children. The proportion of patients with repeat visits was greater for black children (33\%) than white children $(22 \% ; P<0.001)$ and greater for Medicaid-insured $(27 \%)$ than privately insured patients $(21 \% ; P<0.01)$.

Conclusions: This cross-sectional database study demonstrated that black children and those with Medicaid insurance made more ED visits and received somewhat fewer treatments, which may be explained by greater use of the ED for routine care. An opportunity exists for better outpatient management of children with IBD so that nonemergent problems are more effectively handled.
\end{abstract}

Key Words: inflammatory bowel disease, Crohn's disease, pediatric, disparities, race, emergency department

\section{INTRODUCTION}

Racial, ethnic, and socioeconomic disparities have been reported for treatment and outcomes of children and adults with Crohn's disease (CD), ${ }^{1-5}$ a chronic inflammatory bowel disease (IBD) affecting more than 600,000 adults and children in the United States and Canada alone. ${ }^{6}$ According to

Received for publications September 28, 2017; Editorial Decision April 15, 2018. From the *Division of Pediatric Gastroenterology, Hepatology and Nutrition and The Center for Innovation in Pediatric Practice, The Research Institute, Nationwide Children's Hospital, Columbus, Ohio; ${ }^{\dagger}$ University of North Carolina at Chapel Hill, Department of Pediatrics, Division of Gastroenterology and Hepatology, Chapel Hill, North Carolina; ${ }^{\S}$ Biostatistics, College of Public Health, The Ohio State University, Columbus, Ohio

Supported by: Dr. Dotson and this project were supported by the NASPGHAN Foundation/Crohn's and Colitis Foundation of America Young Investigator Development Award. The study sponsors had no role in the study design or the collection, analysis, and interpretation of data.

Conflicts of interest: The remaining authors have no financial relationships or conflicts of interest relevant to this article to disclose.

Address correspondence to: Jennifer L. Dotson, MD, MPH, Nationwide Children's Hospital, 700 Children's Drive, Columbus, OH 43205 (jennifer.dotson@ nationwidechildrens.org). the Institute of Medicine, disparities refer to differences in treatment or access that are not due to differences in patient health status or preference. ${ }^{7}$ Recent public policy initiatives to improve national health care agree that identifying and reducing health-related disparities are essential steps toward that goal. ${ }^{8}$

An especially important area in which to identify potential health care disparities is the emergency department (ED), where multiple diagnostic and treatment decisions are made under stressful conditions. ${ }^{7}$ Under stress and in the absence of standard protocols, human decision-making tends to rely upon cognitive biases such as use of the most recent or most available information or most similar past examples, ${ }^{9,}{ }^{10}$ As such, one might expect disparities in diagnosis and treatment to be more apparent in the ED compared with other health care settings.

The populations under study for disparate treatment are also those who use the ED more frequently. Black, Native American, and Hispanic children are significantly more at risk for recurrent ED visits and high-frequency utilization of EDs. ${ }^{11}$ Patients with Medicaid coverage, low socioeconomic status, and chronic diseases have also been found to utilize ED services more frequently. ${ }^{12}, 13$ There have been similar findings in the IBD population for both adults ${ }^{4}$ and children. ${ }^{2}$ 
Potential disparities in treatment in the ED have been found among adult and pediatric patients. For example, in a recent National Center for Health Statistics report on emergency care in the United States from 2008 to 2010, the mean ED wait time to see a physician for non-Hispanic blacks was 18 minutes longer than for non-Hispanic whites. ${ }^{14}$ A 1-year retrospective, case-cohort study of discharged patients from 2 urban pediatric EDs found that black children were 39\% less likely than non-Hispanic white children to receive any analgesic when presenting to an ED with abdominal pain, and 68\% more likely to have a longer length of stay (LOS).${ }^{15}$ In a large retrospective review of urban pediatric EDs, laboratory testing was ordered almost $40 \%$ less often, and radiological testing 20\% less often, for black than for white children. ${ }^{16}$

As expected, most studies of IBD patients in the ED have focused on adults. A systematic review of studies looking at racial differences in IBD treatment in the ED found no medication differences by race; however, most of these were single- or multicenter and, on average, had fewer than 100 subjects. ${ }^{17}$ In a retrospective review of medical records at a single tertiary care center, black children with IBD were significantly more likely than white children to have a complicated disease course and be treated with corticosteroids and infliximab. ${ }^{3}$ The generalizability of this result is limited by the span of time covered, and the single location and small sample size. It also lacks any measure of family socioeconomic status (SES) to potentially account for identified differences.

At this early stage of the literature, it is difficult to assess to what extent racial and economic disparities exist for the diagnosis and treatment of pediatric IBD patients in the ED. To our knowledge, there are no large, nationally representative studies. To address this gap in the literature, our study aimed to determine the impact of race and insurance status on ED evaluation and treatment among children and adolescents with CD from a large, national administrative database.

Our primary aims were to compare ED medication usage, laboratory and radiological testing, and ED disposition according to race and insurance status. Secondary aims were to determine whether there are race or payor differences in the proportion of patients with repeated visits. Compared with black children, we hypothesized that white children would (1) receive significantly more laboratory and radiologic testing, (2) receive more narcotic pain medications, and (3) have more hospital admissions. We also expected to see a similar trend for those with commercial insurance compared with Medicaid. Finally, the proportion of patients with repeat visits was expected to be greater among black children and those with Medicaid insurance than white and commercially insured children, respectively.

\section{METHODS}

\section{Data Source}

The data for this study were drawn from the Pediatric Health Information System (PHIS), an administrative database containing inpatient, ED, observation, and ambulatory surgery data from 44 not-for-profit tertiary care pediatric hospitals associated with the Children's Hospital Association (CHA; Overland Park, KS, USA). The PHIS hospitals are among the largest children's hospitals in America. Data in PHIS are de-identified upon submission to CHA, but identification numbers allow a patient to be tracked across multiple visits within the same hospital. Both CHA and the individual sites perform data quality and reliability checks (eg, bimonthly coding consensus meetings, coding consistency reviews, and quarterly data quality reports across hospitals) before data are included in the database. This study included data from 38 hospitals, and the total number of hospitals contributing data for a given year varied between 31 and 38 .

\section{Study Design}

This was a retrospective, multicenter, cross-sectional study of pediatric CD patients who had at least $1 \mathrm{ED}$ visit at a participating PHIS center during the study period.

\section{Study Cohort and Visit Criteria}

The unit of analysis for our primary objectives was the ED visit. We examined data for all visits within a 7-year time window (between January 1, 2007, and December 31, 2013) that met the following inclusion criteria: Visits must have been for patients who were $\leq 21$ years old and presented to the ED with either a primary diagnosis of CD (ICD-9 code: 555.x) or a secondary diagnosis of $\mathrm{CD}$ plus a primary CD-related diagnosis. Crohn's disease-related diagnoses (eg, abdominal pain, anemia, extraintestinal manifestations) were determined by author consensus (J.D., W.C., and M.K.) based on a table listing all primary diagnoses among visits with a secondary diagnosis of CD. Generally, the standards of care related to medications and testing studied were consistent across the study period. Additionally, visits must have been for patients who consistently reported being non-Hispanic and either white or black. To maintain strictly defined comparison groups, visits for patients were excluded if race was missing or inconsistently reported, or if patients reported multiracial heritage. As the main analysis is visit-based, payor status could fluctuate for a given individual.

\section{Variables}

Demographics were recorded as follows: age, sex, race, ethnicity, hospital location, date of ED visit, date of discharge, insurance payor status, encrypted ZIP code, discharge ID, primary and secondary discharge diagnoses, and median neighborhood income. Median neighborhood income was derived by the PHIS data analytics team and was based on the 2010 US Census data referring to actual patient ZIP codes reported at index hospitalization. As PHIS hospitals transitioned to a new reporting format for race and payor status at different times between 2007 and 2009, coding algorithms were used to convert old and new versions of these variables 
into a form that accurately reflects information from both versions. Insurance payor status was coded as Medicaid or private insurance (Appendix A), and race was coded as black or white.

Additional variables included the following: medications administered (eg, corticosteroids, antibiotics, pain medications, anti-emetics, and "other," indicating all other medications that do not belong to 1 of the aforementioned categories), ED disposition (eg, admitted to inpatient status, discharged, transferred), laboratory testing (eg, chemistries, immunology), imaging studies (eg, magnetic resonance imaging [MRI], ultrasound, computed tomography [CT], x-rays), and number of visits within the study time frame.

\section{Outcomes}

The primary outcomes were medication usage, laboratory and radiological testing, and patient disposition. The secondary outcome was the number of ED visits per unique patient. Primary outcomes were compared in terms of race (non-Hispanic black vs non-Hispanic white) and insurance payor status (private vs Medicaid).

\section{Statistical Analyses}

Demographic characteristics were compared between white and black patients using patient-level data. Pearson chisquare tests were used for categorical characteristics (sex, geographic region, insurance payor status, and income quartile) and to compare the proportion of repeat vs nonrepeat ED visits by race and by payor status. A 2-sample $t$ test was used for age at first visit during the study period, and a nonparametric Kruskal-Wallis rank-sum test was used for the number of ED visits per patient.

Mixed-level logistic regression modeling was used to investigate associations between the predictors (race and payor status, in separate models) and primary visit-level outcomes (medication usage, radiological and laboratory testing, and disposition). Fixed effects included race (and/or payor status), and random effects included subject and hospital. The subject-specific intercept controls for within-subject correlation (eg, patients with multiple visits), and hospital location controls for site-specific effects (eg, treatment protocols that may differ by hospital; racial diversity of the local patient population). In addition, for outcomes where there was a statistically significant fixed effect for race or for payor status, a bivariate model with both race and payor status was used to determine whether each effect remained significant after controlling for the other fixed effect. In post hoc analyses for significant unadjusted effects of race or payor status, an interaction model was tested using race, payor status, and their interaction. To look for stratification of race by payor status, this was followed by a model with a single fixed effect for race, which was run with (a) Medicaid only visits and then (b) commercial only visits.
Models were fit using the glmer command from the lme4 package, ${ }^{18} \mathrm{v} 1.1-7$, in R 3.0.1. ${ }^{19}$ Models that failed to converge were retested with recommended trouble-shooting techniques ${ }^{18}$ such as increasing the number of iterations and employing alternative nonlinear optimizing algorithms for estimating the variance-covariance matrix of random effects. With continued nonconvergence, a simpler model was chosen, which included the fixed effect (of race or payor status) and either (1) the random effect for hospital or (2) the random effect for patientdepending on convergence and the Bayesian Information Criterion. So that models would be comparable, the random effects component of each bivariate model was the same as that for the corresponding single fixed effect model.

A $P$ value of $<0.05$ was considered statistically significant. This study was approved by the Nationwide Children's Hospital Institutional Review Board.

\section{RESULTS}

\section{Demographics}

The data included in this study resulted from 3779 ED visits, representing 2618 patients (23\% black, 77\% white) with an approximately equal sex distribution (Fig. 1). Table 1 summarizes the subject-based demographics according to race/ethnicity for each patient. There were several differences between the cohorts with respect to geographic region, payor status, and median income. Black children were more likely to have Medicaid insurance $(P<0.001)$ and a lower median neighborhood income $(P<0.001)$.

\section{Medication Usage}

Emergency department visits for white children with CD had a higher odds of receiving any medication (OR, 1.50; 95\% CI, 1.15-1.96), anti-emetics (OR, 1.72; 95\% CI, 1.26-2.34), and other drugs (OR, 1.67; 95\% CI, 1.31-2.14) compared with black children. Visits for those who were privately insured had a higher odds of receiving anti-emetics (OR, 1.43; 95\% CI, 1.051.93) and other drugs (OR, 1.42; 95\% CI, 1.10-1.84) compared with those who were Medicaid insured (Table 2). When controlling for payor status, the odds ratios related to race declined. The odds ratio for any drug became only marginally significant (OR, 1.36; 95\% CI, 1.00-1.86), whereas the odds ratios for other drugs (OR, 1.50; 95\% CI, 1.12-2.01) and for anti-emetics (OR, 1.52; 95\% CI, 1.06-2.17) remained significant. When controlling for race, odds ratios relating to payor effects were no longer significant.

\section{Laboratory Evaluation}

Emergency department visits for white children had a higher odds of having any laboratory test (OR, 1.57; 95\% CI, 1.17-2.11), a complete blood count (CBC; OR, 1.41; 95\% CI, 1.09-1.81), chemistries (OR, 1.47; 95\% CI, 1.14-1.89), and $\mathrm{C}$-reative protein (CRP)/erythrocyte sedimentation rate (ESR; 


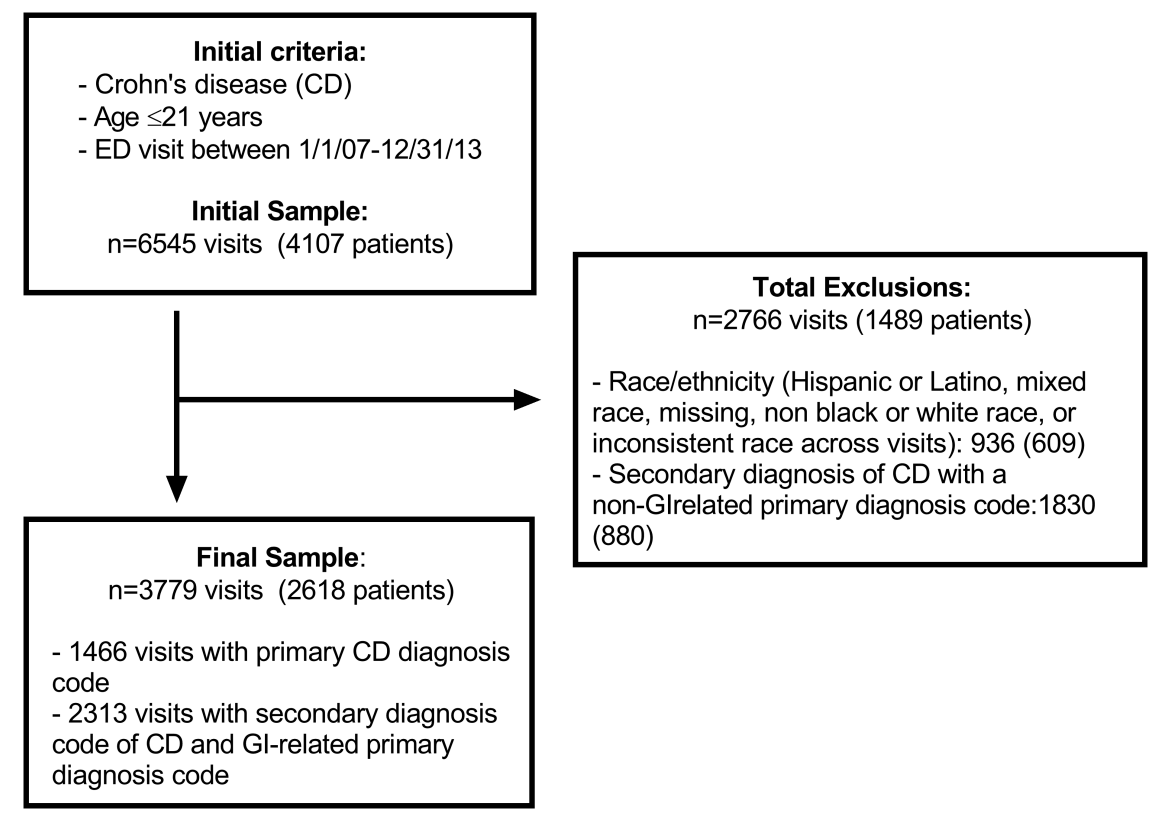

Figure 1. Flowchart for the final cohort selection and exclusion criteria. After applying exclusion criteria to the initial database sample of patients with Crohn's disease age $\leq 21$ years with an ED visit between 2007 and 2013, and subsequently applying race/ethnicity exclusions, the final study cohort was determined. Abbreviation: Gl, gastrointestinal.

OR, $1.39 ; 95 \%$ CI, 1.08-1.78) as part of the evaluation compared with black children. Similarly, visits for those who were privately insured had a higher odds of having any laboratory test (OR, 1.43; 95\% CI, 1.04-1.97), a complete blood count (OR, 1.51; 95\% CI, 1.16-1.98), chemistries (OR, 1.36; 95\% CI, 1.05-1.77), and CRP/ESR (OR, 1.47; 95\% CI, 1.14-1.89) (Table 3). However, after controlling for payor status, odds ratios based on race were diminished, and none remained significant. When controlling for race, odds ratios based on payor status remained significant for $\mathrm{CBC}(\mathrm{OR}, 1.43$; $95 \% \mathrm{CI}, 1.08$ $1.90)$ and for CRP/ESR (OR, 1.39; 95\% CI, 1.06-1.82).

\section{Radiologic Imaging}

Emergency department visits for white children had a higher odds of having any imaging (OR, 1.44; 95\% CI, 1.151.80) and a CT scan (OR, 2.12; 95\% CI, 1.36-3.31) compared with black children. Visits for those with private insurance had a higher odds of receiving any radiologic imaging (OR, 1.35; 95\% CI, 1.08-1.70), x-ray (OR, 1.29; 95\% CI, 1.02-1.63), and CT scan (OR, 1.67; 95\% CI, 1.06-2.61) compared with those with Medicaid insurance (Table 3). For each imaging outcome, the odds ratio for race was diminished and became nonsignificant after controlling for payor status and race.

\section{ED Repeat Visit Frequency and Disposition}

When evaluating a cohort of patients with more than 1 ED visit within the 7-year study period, there was a higher proportion of black children $(33 \%$ vs $22 \% ; P<0.001)$ and those with Medicaid insurance ( $27 \%$ vs $21 \% ; P<0.01)$ compared with white children and private insurance, respectively
(Table 4).There were no apparent differences by race or payor status in the percentage of visits where patients were admitted, discharged, or transferred following ED visits. Of note, visits involving private insurance were less likely than those involving Medicaid to have missing disposition data (OR, 0.34; 95\% CI, $0.17-0.66$ ).

\section{Post Hoc Analyses}

In post hoc analysis of the significant unadjusted outcomes in Tables 2 and 3, there was a significant interaction between race and payor status for 4 separate laboratory outcomes (any lab, CBC, chemistries, and CRP/ESR). To explore the nature of these interactions, we evaluated the effects of race stratified by payor status. For each lab outcome, there was a small effect of race in the Medicaid population and no effect of race in the commercial payor population. (Table 5)

\section{DISCUSSION}

In a comprehensive evaluation of pediatric IBD patients who use the ED, adjusted analyses (with minor exceptions) found no significant racial or insurance-related differences in the use of imaging, laboratory testing, and medications. Contrary to expectations, white children were no more likely to receive narcotic analgesics. By contrast, in a large, nationally representative sample of pediatric patients in the ED with abdominal pain, Johnson et al. ${ }^{15}$ found small- to medium-sized effects, with whites being 1.6 times more likely than blacks to receive any analgesic and 2.6 times more likely to receive a narcotic analgesic. A key difference may be that the prior study included subjects whose primary complaint was abdominal 
TABLE 1: Demographic Characteristics of Emergency Department Visits by Race for a Pediatric Crohn's Disease Cohort

\begin{tabular}{|c|c|c|c|c|}
\hline \multirow[b]{2}{*}{ Variable } & \multirow[b]{2}{*}{ Total } & \multicolumn{2}{|c|}{ Subject-Based } & \multirow[b]{2}{*}{$P$} \\
\hline & & $\begin{array}{c}\text { NH Black } \\
\text { No. }(\%)\end{array}$ & $\begin{array}{c}\text { NH White } \\
\text { No. }(\%)\end{array}$ & \\
\hline Total & 2618 & $612(23)$ & $2006(77)$ & \\
\hline Male & $1312(50)$ & $297(49)$ & $1015(51)$ & $>0.99$ \\
\hline Age-1st visit, mean (SD), y & $13.97(4.00)$ & $13.86(4.30)$ & $14.00(3.90)$ & $>0.99$ \\
\hline Region $^{\mathrm{a}}$ & & & & $<0.001$ \\
\hline Midwest & $891(34)$ & $211(34)$ & $680(34)$ & \\
\hline Northeast & $559(21)$ & $95(16)$ & $464(23)$ & \\
\hline South & $877(33)$ & $271(44)$ & $606(30)$ & \\
\hline West & $291(11)$ & $35(6)$ & $256(13)$ & \\
\hline Payor $^{\mathrm{b}}$ & & & & $<0.001$ \\
\hline Private & $1331(51)$ & $186(30)$ & $1145(57)$ & \\
\hline Medicaid & $612(23)$ & $261(43)$ & $351(17)$ & \\
\hline Other & $374(14)$ & $100(16)$ & $274(14)$ & \\
\hline Missing & $301(11)$ & $65(11)$ & $236(12)$ & \\
\hline Median income ${ }^{\mathrm{c}}$ & & & & $<0.001$ \\
\hline Q1: \$11,130-\$35,562 & $493(23)$ & $196(42)$ & $297(17)$ & \\
\hline Q2: \$35,564-\$45,666 & $534(24)$ & $117(25)$ & $417(24)$ & \\
\hline Q3: \$45,730-\$59,671 & $581(27)$ & $81(17)$ & $500(29)$ & \\
\hline Q4: \$59,718-\$148,263 & 577 (26) & $69(15)$ & $433(30)$ & \\
\hline
\end{tabular}

All percentages are by column. Subjects are counted by index visit. Bolded $P$ values represent significant findings.

Abbreviation: $\mathrm{NH}$, non-Hispanic.

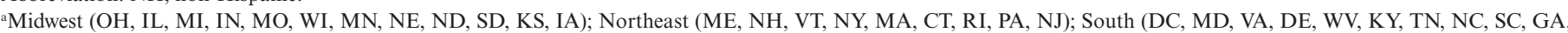
AL, MS, FL, LA, TX, AR, OK); West (AZ, CO, CA, WA, OR, MT, WY, ID, NV, UT, NM, HI, AK).

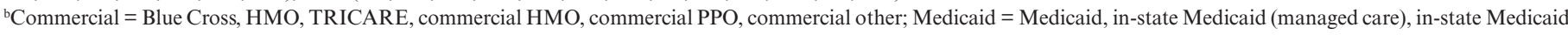

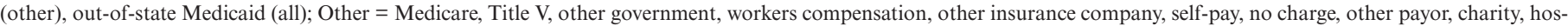
pital chose not to bill; Missing = not recorded, invalid code, unknown.

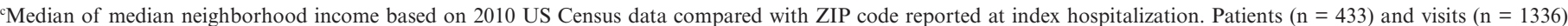
missing on income are excluded. Visit-based income quartiles differ at Q2 as follows: Q2 $(\$ 35,564-\$ 45,706)$.

pain, cramps, or spasms, whereas abdominal pain was only 1 of several secondary CD-related diagnoses in the present study. The present study may also have been underpowered to identify analgesic treatment differences by race.

In adjusted analyses, white children were just as likely to receive any imaging overall, and those with private insurance, compared with public insurance, were as likely to receive any imaging, and $\mathrm{x}$-ray and CT scans. By contrast, a previous study found that white patients were more likely than Englishspeaking black patients to receive any imaging (adjusted OR, 1.18; 95\% CI, 1.05-1.32). ${ }^{16}$ Although the present study looked at all visits, the prior study restricted its focus to patient diagnoses with a greater likelihood for imaging services (ie, asthma, fever, or acute respiratory tract infection).

The finding that black patients and those with Medicaid had more repeat visits to the ED within the study period could indicate greater use of the ED for more routine, less urgent care and receiving fewer services, although this is speculative without definitive information on disease severity at presentation.
Previous studies have found that those with Medicaid are more likely than those with private insurance to use the ED for routine care, ${ }^{20,21}$ and this may be due to a variety of factors including convenience (ie, scheduling and proximity) and lack of copays. ${ }^{22,23}$

Patient cost-sharing ${ }^{24,} 25$ and education have been proposed as potential solutions to better manage outpatient and emergency care. Copays are effectively used by private insurers to control costs and disincentivize the use of the ED for nonurgent care. ${ }^{24,25}$ For example, prior literature consistently finds that copayments reduce the use of medications, ${ }^{26}$ with lower-income patients being more sensitive to cost. For ED visits, Selby et al. $^{27}$ found a greater reduction among low-SES neighborhoods $(22.5 \%)$ compared with all other neighborhoods $(14.6 \%)$ after the introduction of moderate copays of \$25-\$30 in a large HMO. Slightly larger reductions in ED visits $(26 \%)$ were found with more substantial copayments of $\$ 50-\$ 100$; however, these results were not stratified by visit severity. ${ }^{28}$ Those with low income and/or chronic conditions may be especially vulnerable 


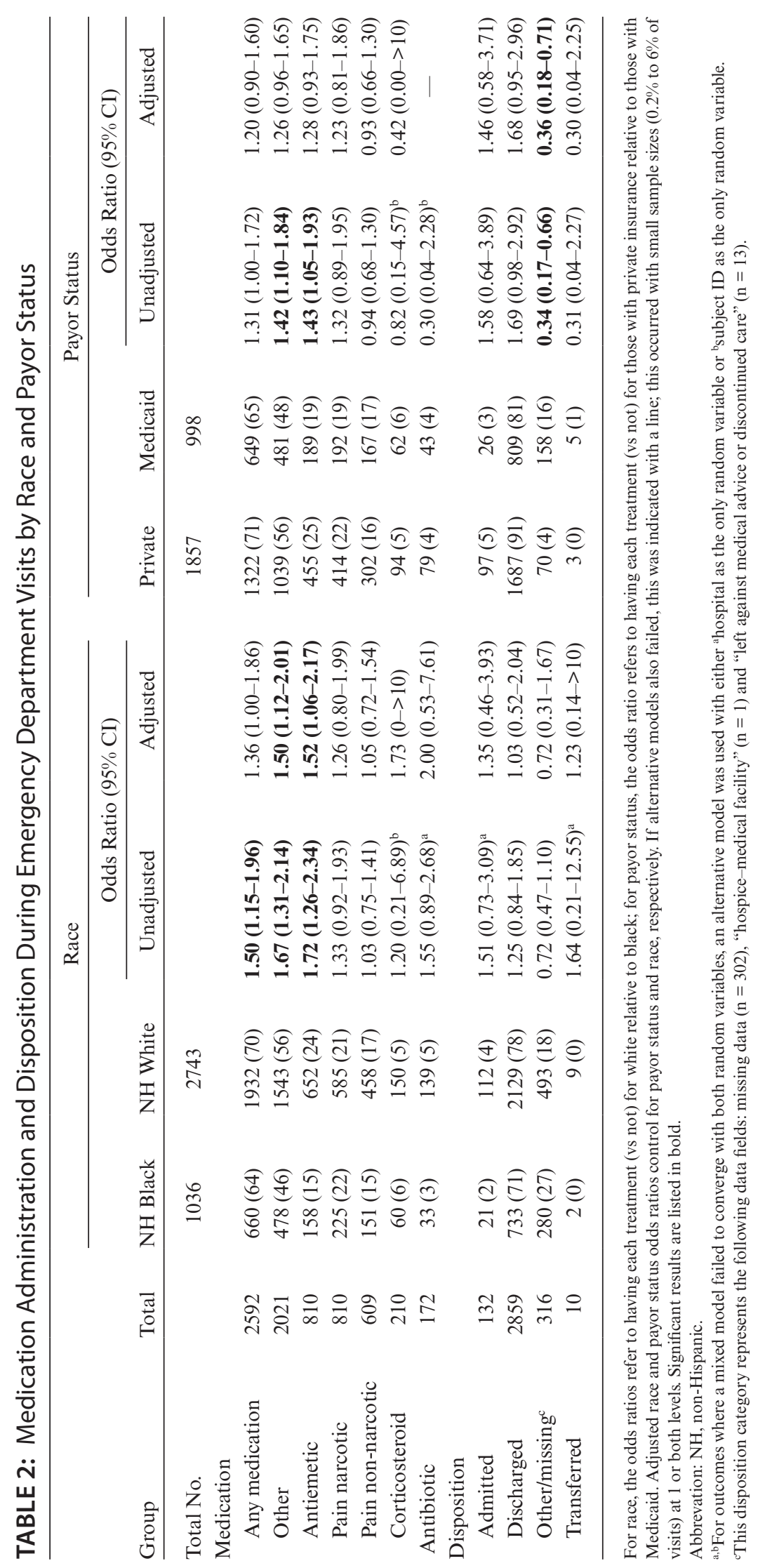




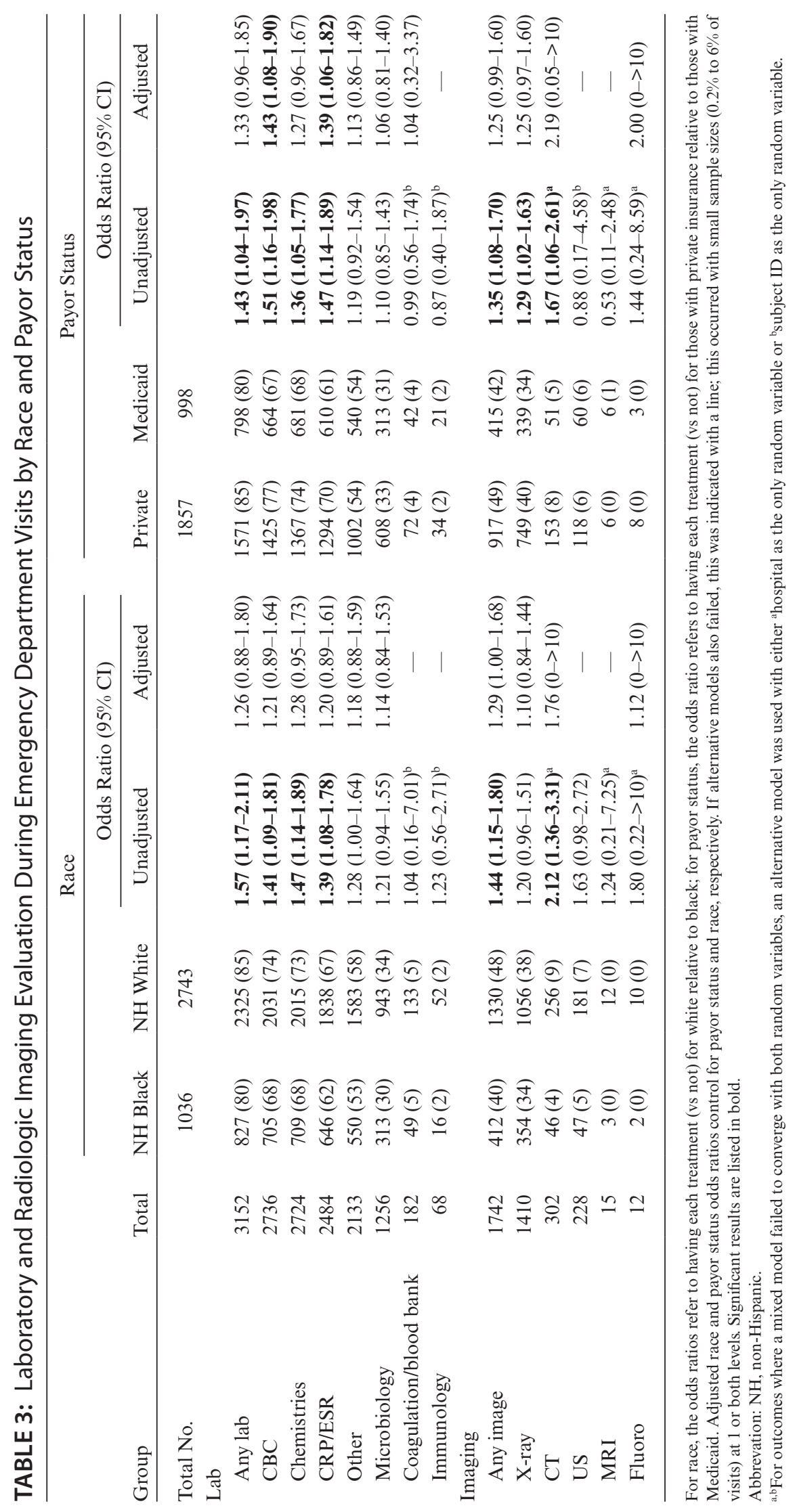


TABLE 4: Emergency Department Visits According to Patient Race/Ethnicity and Payor Status Over the 7-Year Study Period

\begin{tabular}{|c|c|c|c|c|c|c|c|}
\hline & \multicolumn{4}{|c|}{ Patients by Race/Ethnicity } & \multicolumn{3}{|c|}{ Patients by Payor Status } \\
\hline & Total & $\mathrm{NH}^{\mathrm{a}}$ Black & NH White & $P$ & Private & Medicaid & $P$ \\
\hline Total No. visits & 3779 & 1036 & 2743 & - & 1857 & 998 & - \\
\hline Total No. patients & 2618 & 612 & 2006 & - & 1392 & 661 & - \\
\hline Visits/patient, median (IQR) & $1(1-1)$ & $1(1-1)$ & $1(1-1)$ & $>0.99$ & $1(1-1)$ & $1(1-2)$ & $>0.99$ \\
\hline Patients $=1$ visit, No. $(\%)$ & $1983(76)$ & $411(67)$ & $1572(78)$ & & $1101(79)$ & $485(73)$ & \\
\hline a,cPatients >1 visit, No. $(\%)$ & $635(24)$ & $201(33)$ & $434(22)$ & $<0.001$ & $291(21)$ & $176(27)$ & $<0.01$ \\
\hline${ }^{\mathrm{b}, \mathrm{c}}$ Patients $>3$ visits, No. $(\%)$ & $105(5)$ & $39(9)$ & $66(4)$ & $<0.001$ & $38(3)$ & $31(6)$ & $<0.05$ \\
\hline
\end{tabular}

Bolded $P$ values represent significant findings.

Abbrevation: NH, non-Hispanic.

${ }^{a}$ Chi-square comparisons: repeat ED visitor (1 visit vs $>1$ visit $) \times(1)$ race and (2) payor status

${ }^{\mathrm{b}}$ Chi-square comparisons: repeat ED visitor (1 visit vs $>3$ visits) $\times(1)$ race and (2) payor status

cPercentages are a function of the number of patients included in the chi-square, which may

or may not sum to the total number of patients.

TABLE 5: Post Hoc Interaction and Stratified Models for Significant Outcomes

\begin{tabular}{|c|c|c|c|}
\hline \multicolumn{4}{|c|}{ Interaction Model, OR $(95 \% \mathrm{CI})^{\mathrm{a}}$} \\
\hline & Race & Payor & Race $\times$ Payor \\
\hline Any lab & $1.82(1.11-2.98)$ & $2.29(1.25-4.22)$ & $0.45(0.22-0.93)$ \\
\hline $\mathrm{CBC}$ & $1.76(1.15-2.68)$ & $2.51(1.49-4.25)$ & $0.44(0.24-0.82)$ \\
\hline Chemistries & $2.07(1.36-3.17)$ & $2.52(1.51-4.20)$ & $0.37(0.20-0.67)$ \\
\hline CRP/ESR & $1.84(1.22-2.78)$ & $2.59(1.57-4.28)$ & $0.41(0.23-0.74)$ \\
\hline \multicolumn{4}{|c|}{ Race stratified by payor status ${ }^{\mathrm{b}}$} \\
\hline \multicolumn{4}{|c|}{ Race OR $(95 \% \mathrm{CI})$} \\
\hline & Medicaid only & Commercial only & \\
\hline Any lab & $1.79(1.05-3.05)$ & $0.81(0.46-1.42)$ & \\
\hline $\mathrm{CBC}$ & $1.77(1.14-2.74)$ & $0.78(0.49-1.25)$ & \\
\hline Chemistries & $2.02(1.31-3.10)$ & $0.75(0.47-1.19)$ & \\
\hline CRP/ESR & $1.80(1.19-2.73)$ & $0.74(0.47-1.15)$ & \\
\hline
\end{tabular}

Significant odds ratios listed in bold.

${ }^{a}$ The interaction model for each outcome includes race, payor status, and race $\times$ payor status fixed effects plus hospital and patient medical record number as random effects

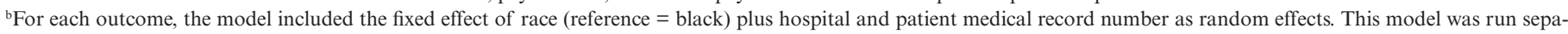
rately for visits with Medicaid and commercial payor status.

to "cost-offset" effects. Incentivizing patients to delay care until their condition is more severe could lead to more expensive care for the same episode, though this may not be true for child visits. ${ }^{29}$

Parent medical knowledge may be unreliable to determine the appropriate use of the $\mathrm{ED}^{30}$; however, interventions involving patient or parent education can be effective. Sturm et al. ${ }^{23}$ encouraged families with children to use their primary care physicians for less urgent episodes over a 12-month period through a program that developed medical decision guidelines for afterhours care, outlined primary care provider services and availability, and sometimes included access to physician advice after hours by phone. In this study, 12\% fewer families in the intervention used ED services for nonurgent episodes compared with controls.

There were several important limitations in this study. One of the biggest limitations is the lack of detailed information regarding the characteristics of the source population from which patients were drawn at each hospital location. Thus, we cannot determine how many people of each race and payor 
status had 0 visits to derive an accurate proportion of total visits for each subgroup. Questions regarding which groups use the ED more frequently are therefore beyond the scope of the current study. We evaluated ED visits within PHIS-associated hospitals, and this does not account for differences in outpatient management that are not captured by PHIS. Approximately $8 \%$ of ED visits were missing the disposition field. As this disproportionately affected those with Medicaid insurance, results for low-frequency dispositions (admitted and transferred visits) may be less reliable. The PHIS database is not weighted for extrapolation to national estimates; thus results may not be generalizable to all pediatric centers. This was a retrospective study based on administrative data, so reporting bias or classification errors may be present, although there are data quality measures in place to minimize errors. As the findings were not based on detailed medical record review, we were unable to assess or control for disease presentation or disease severity. Finally, although we have interpreted treatment differences by race as an underuse of services among black patients, they might represent overuse among white patients. The absence of a clear guideline or reference point for expected treatment in each case renders it difficult to distinguish between these 2 interpretations. The strengths of the study include its large, regionally diverse sample population of ED visits and the use of an analytical method that appropriately accounted for data clustering by patient and hospital.

In summary, several small ${ }^{31}$ treatment differences were identified separately by race and by insurance payor status in a large and diverse cohort of pediatric patients with $\mathrm{CD}$ visits to the ED. Given the strong overlap between race and insurance type with white patients more likely to have private insurance and black patients more likely to have Medicaid, it is not surprising that, for several treatment outcomes, racial differences were no longer apparent after accounting for payor differences, despite expectations based on the literature. To the extent that the 2 predictors are correlated, the effective sample size is reduced, and it is often difficult to tease out their independent effects. Although effect sizes identified in this study were small, it is important to minimize such differences as much as possible. Interestingly, in the post hoc analyses, white patients were more likely to receive any lab testing when using Medicaid but not private insurance. It seems that being black and having limited access to insurance represents a double risk in terms of access to some care. Perhaps white patients were treated the same irrespective of insurance. These differences may represent differences between hospitals (eg, black patients in some urban areas may use hospitals with lower rates of evidence-based practices ${ }^{32}$ or limited resources).

The root causes of health care disparities in the United States are complex and beyond the scope of this study, which is limited to identifying racial and economic disparities. Given the limitations of our data set, we cannot determine whether these small apparent differences represent racial disparities in the quality of health care. In general, multiple strategies may be necessary to address the inappropriate use of the ED, especially among families with lower incomes. Our results suggest an opportunity for better outpatient management of children with IBD, particularly among black children and those with Medicaid, so that nonemergent problems are more effectively handled in the office setting rather than ED. Examples of interventions to improve our outpatient management approach include extended evening and weekend clinic hours, enhancement and utilization of electronic patient portals, increasing the focus on proactive management of these patients (eg, use of clinical dashboards to monitor from a population perspective), providing additional education and resources to our patients and families, and fully integrating supportive multidisciplinary teams - including case managers, social workers, and psychologists - into our practice. Addressing these complex psychosocial issues and social determinants of health is particularly challenging without embedded social workers and psychologists. Large, carefully crafted prospective studies with a focus on social determinants of health are needed to address these issues.

In addition, future research should focus on how to address family concerns about inconvenience surrounding primary care or specialty outpatient clinics and the effective use of copays without discouraging ED services when necessary.

\section{ACKNOWLEDGMENTS}

We gratefully acknowledge John Burian, MS, part of Dr. Veronica Vieland's lab at the Battelle Center for Mathematical Medicine in the Research Center at Nationwide Children's Hospital, for his time and effort in running preliminary analyses.

\section{REFERENCES}

1. Sewell JL, Velayos FS. Systematic review: the role of race and socioeconomic factors on IBD healthcare delivery and effectiveness. Inflamm Bowel Dis. 2013;19:627-643.

2. Benchimol EI, To T, Griffiths AM, et al. Outcomes of pediatric inflammatory bowel disease: socioeconomic status disparity in a universal-access healthcare system. J Pediatr. 2011;158:960-967.e1.

3. Eidelwein AP, Thompson R, Fiorino K, et al. Disease presentation and clinica course in black and white children with inflammatory bowel disease. $J$ Pediatr Gastroenterol Nutr. 2007;44:555-560.

4. Nguyen GC, LaVeist TA, Harris ML, et al. Racial disparities in utilization of specialist care and medications in inflammatory bowel disease. Am J Gastroenterol. 2010;105:2202-2208.

5. Dotson JL, Kappelman MD, Chisolm DJ, et al. Racial disparities in readmission, complications, and procedures in children with Crohn's disease. Inflamm Bowel Dis. 2015;21:801-808.

6. Loftus EV Jr. Clinical epidemiology of inflammatory bowel disease: incidence, prevalence, and environmental influences. Gastroenterology. 2004;126:1504-1517.

7. Institute of Medicine. Unequal Treatment: Confronting Racial and Ethnic Disparities in Health Care. Washington, DC: The National Academies Press; 2003.

8. Shi L, Stevens GD. Disparities in access to care and satisfaction among U.S. children: the roles of race/ethnicity and poverty status. Public Health Rep. 2005;120:431-441.

9. Elstein AS. Heuristics and biases: selected errors in clinical reasoning. Acad Med. 1999;74:791-794.

10. Phua DH, Tan NC. Cognitive aspect of diagnostic errors. Ann Acad Med Singapore. 2013;42:33-41.

11. Alpern ER, Clark AE, Alessandrini EA, et al; Pediatric Emergency Care Applied Research Network (PECARN). Recurrent and high-frequency use of the emergency department by pediatric patients. Acad Emerg Med. 2014;21:365-373. 
12. Vinton DT, Capp R, Rooks SP, et al. Frequent users of US emergency departments: characteristics and opportunities for intervention. Emerg Med J. 2014;31:526-532.

13. Hunt KA, Weber EJ, Showstack JA, et al. Characteristics of frequent users of emergency departments. Ann Emerg Med. 2006;48:1-8.

14. National Center for Health Statistics. Health, United States, 2012: With Special Feature on Emergency Care. Hyattsville, MD; 2013.

15. Johnson TJ, Weaver MD, Borrero S, et al. Association of race and ethnicity with management of abdominal pain in the emergency department. Pediatrics. 2013:132:e851-e858.

16. Payne NR, Puumala SE. Racial disparities in ordering laboratory and radiology tests for pediatric patients in the emergency department. Pediatr Emerg Care. 2013;29:598-606.

17. Afzali A, Cross RK. Racial and ethnic minorities with inflammatory bowel disease in the United States: a systematic review of disease characteristics and differences. Inflamm Bowel Dis. 2016;22:2023-2040.

18. Bates D, Mächler M, Bolker B, et al. Fitting linear mixed-effects models using lme4. J Stat Softw. 2015;67:48.

19. $R$ 3.0.1 [computer program]. Vienna, Austria: R Foundation for Statistical Computing; 2013.

20. Phelps K, Taylor C, Kimmel S, et al. Factors associated with emergency department utilization for nonurgent pediatric problems. Arch Fam Med. 2000;9:1086-1092.

21. Taubman SL, Allen HL, Wright BJ, et al. Medicaid increases emergency-department use: evidence from Oregon's health insurance experiment. Science. 2014;343:263-268

22. Berry A, Brousseau D, Brotanek JM, et al. Why do parents bring children to the emergency department for nonurgent conditions? A qualitative study. Ambul Pediatr. 2008;8:360-367.
23. Sturm JJ, Hirsh D, Weselman B, et al. Reconnecting patients with their primary care provider: an intervention for reducing nonurgent pediatric emergency department visits. Clin Pediatr (Phila). 2014;53:988-994.

24. Reed M, Fung V, Brand R, et al. Care-seeking behavior in response to emergency department copayments. Med Care. 2005;43:810-816.

25. Wallace NT, McConnell KJ, Gallia CA, et al. How effective are copayments in reducing expenditures for low-income adult Medicaid beneficiaries? Experience from the Oregon Health Plan. Health Serv Res. 2008;43:515-530.

26. Lexchin J, Grootendorst P. Effects of prescription drug user fees on drug and health services use and on health status in vulnerable populations: a systematic review of the evidence. Int J Health Serv. 2004;34:101-122.

27. Selby JV, Fireman BH, Swain BE. Effect of a copayment on use of the emergency department in a health maintenance organization. $N$ Engl $\mathrm{J}$ Med. 1996;334:635-641.

28. Hsu J, Price M, Brand R, et al. Cost-sharing for emergency care and unfavorable clinical events: findings from the safety and financial ramifications of ED copayments study. Health Serv Res. 2006;41:1801-1820.

29. Wharam JF, Zhang F, Landon BE, et al. Low-socioeconomic-status enrollees in high-deductible plans reduced high-severity emergency care. Health Aff (Millwood). 2013;32:1398-1406.

30. Diekema DS, Del Beccaro MA, Cummings P, Quan L. Physician parents and utilization of a pediatric emergency department. Pediatr Emerg Care. 1996;12:400-403.

31. Chen HN, Cohen P, Chen S. How big is a big odds ratio? Interpreting the magnitudes of odds ratios in epidemiological studies. Commun Stat-Simul C. 2010;39:860-864.

32. Barnato AE, Lucas FL, Staiger D, et al. Hospital-level racial disparities in acute yocardial infarction treatment and outcomes. Med Care. $2005 ; 43: 308-319$

\section{APPENDIX A: Mapping Key for Each Payor Category According to PHIS Payor Titles from Version 1 and Version 2}

\begin{tabular}{|c|c|c|}
\hline \multirow[b]{2}{*}{ Payor Categories } & \multicolumn{2}{|c|}{ PHIS Payor Title } \\
\hline & Version $1^{\text {a }}$ & Version $2^{\mathrm{a}}$ \\
\hline Medicaid & Medicaid & $\begin{array}{l}\text { In-state Medicaid (managed care); in-state Medicaid } \\
\text { (other), out-of-state Medicaid (all) }\end{array}$ \\
\hline Commercial & HMO, Blue Cross & Commercial (HMO, PPO, or other), TRICARE \\
\hline Other & $\begin{array}{l}\text { Medicare, other government, self-pay, other insurance company, no } \\
\text { charge, Title V, workers compensation, other }\end{array}$ & $\begin{array}{l}\text { Medicare, Other government, self-pay, other payor, } \\
\text { hospital chose not to bill, charity }\end{array}$ \\
\hline Missing & Not recorded, invalid & Not recorded, invalid, unknown \\
\hline
\end{tabular}

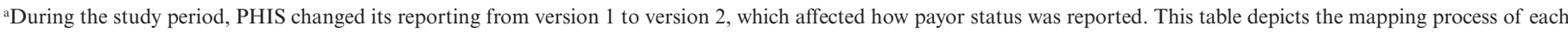
version into the 3 categories we used for analysis. 